\title{
Lead externalization - a potential source of lead-related infective endocarditis?
}

\author{
Maciej Polewczyk ${ }^{1}$, Dorota Nowosielecka², Konrad Tomków³ ${ }^{3}$ Łukasz Tułecki ${ }^{3}$, Andrzej Kutarski ${ }^{4}$, \\ Anna Polewczyk ${ }^{1}$ \\ ${ }^{1}$ Institute of Medical Sciences, Jan Kochanowski University, Kielce, Poland \\ ${ }^{2}$ Department of Cardiology, The Pope John Paul II Province Hospital, Zamosc, Poland \\ ${ }^{3}$ Department of Cardiac Surgery, The Pope John Paul II Province Hospital, Zamosc, Poland \\ ${ }^{4}$ Department of Cardiology, Medical University of Lublin, Lublin, Poland
}

Adv Interv Cardiol 2018; 14, 3 (53): 316-317

DOI: https://doi.org/10.5114/aic.2018.78340

A 32-year-old patient with a single chamber implantable cardioverter-defibrillator (ICD) was admitted to a cardiological ward due to recurrent episodes of fever since 1 month before. Blood tests showed a slightly elevated C-reactive protein (CRP) level without leukocytosis or procalcitonin increase. The transthoracic echocardiography (TTE) examination revealed a vegetation $(29 \mathrm{~mm} \times 6 \mathrm{~mm})$ in the lower right atrium connected with the lead; the image was confirmed in transesophageal echocardiography (TEE). Negative blood culture was only probably negative due to the antibiotic therapy. The patient was diagnosed with lead-related infective endocarditis (LRIE), received modified antibiotic therapy and was initially qualified for the surgical extraction of the ICD system, but he did not agree to surgery. After consultation with the referential transvenous lead extraction (TLE) center the patient was qualified for TLE. Preoperative fluoroscopy revealed an interesting finding - ICD lead externalization was observed at the same location as the previously described vegetation. The TLE procedure was performed under general anesthesia in a hybrid operating room with intraoperative TEE monitoring and cardiac surgical standby. Due to the presence of a relatively large vegetation, a special nitinol basket was introduced into the main pulmonary artery for pulmonary circulation protection. The lead was removed using a Byrd dilator sheath (Cook, USA). Although during the procedure the vegetation was fragmented (only small parts were caught by the basket) there were no clinical signs of pulmonary embolism. There were no periprocedural complications. Postoperative examination of the extracted Riata (St. Jude Med- ical, USA) defibrillating lead showed significant cable externalization with remnants of the vegetation and relevant lead degeneration (Figure 1 ).

Lead externalization is relatively common among Riata leads. However, in the literature we may find cases of such a phenomenon in different leads produced by different manufacturers [1]. The mechanism of externalization of Riata leads is related to their specific construction. Inside there are several conductors which may move relative to the insulation, leading to its further damage - 'inside-out abrasion'. In some research there was no evident correlation between lead externalization and electrical failure [2], but a large Canadian registry confirmed the impact of externalization on lead failure [3]; in our case the electrical parameters of the ICD system remained stable. The influence of lead externalization on LRIE development has not been investigated yet; there are only a few similar reports [4]. An externalized cable might be thrombogenic. A thrombus formation around the location of externalization may become the origin of a further infection. According to the recent reports, some patients with vegetations even exceeding $4 \mathrm{~cm}$ in diameter underwent TLE, avoiding cardiac surgery. However, the decision of the extraction method was made individually considering the total vegetation volume, the degree of infiltration of heart structures and the real risk of severe pulmonary embolism [5]. In clinical practice the decision of removal of a functional lead with present externalization remains problematic. Apart from possible electrical failure one should have in mind the fact that exposed internal lead conductors might be the chief sites of serious infection.

\section{Corresponding author:}

Maciej Polewczyk MD, The Institute of Medical Sciences, Jan Kochanowski University, 5 Żeromskiego St, 27-369 Kielce, Poland, phone: +48534 461 019, e-mail: maciek.polewczyk@gmail.com

Received: 23.02.2018, accepted: 17.04.2018. 

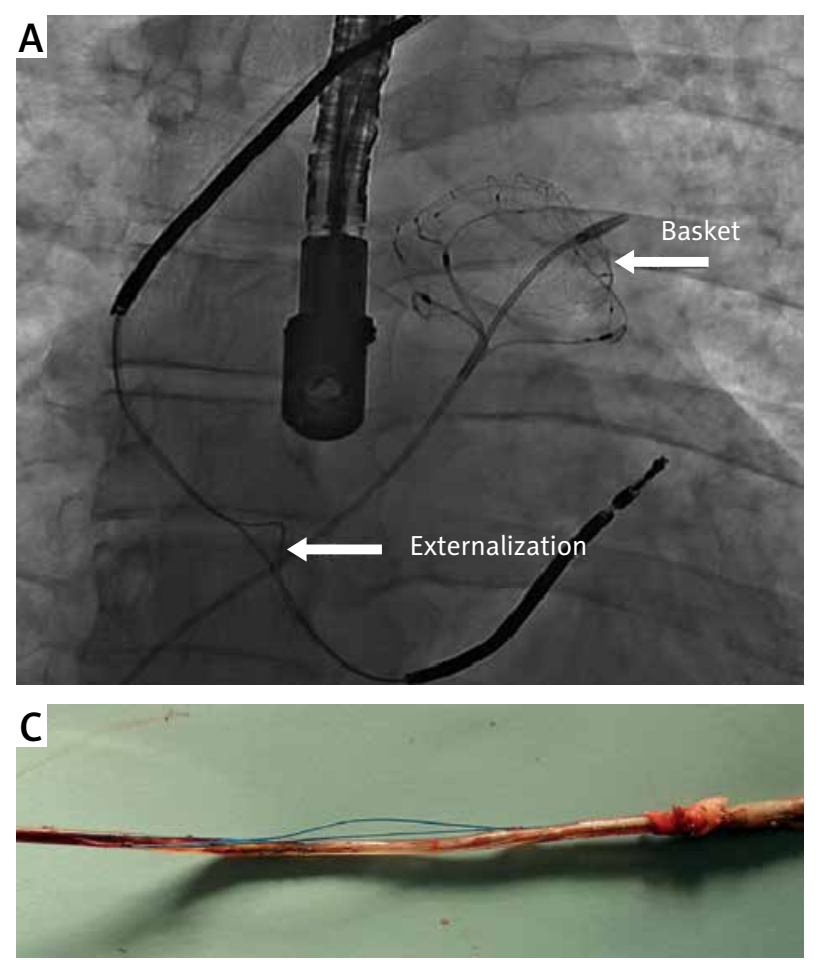

\section{Conflict of interest}

The authors declare no conflict of interest.

\section{References}

1. De Maria E, Borghi A, Bonetti L et al. Externalized conductors and insulation failure in Biotronik defibrillator leads: history repeating or a false alarm? World J Clin Cases 2017; 5: 27-34.

2. Demirel F, Adiyaman A, Delnoy PP, et al. Mechanical and electrical dysfunction of Riata implantable cardioverter-defibrillator leads. Europace 2014; 16: 1787-94.

3. Parkash R, Thibault B, Mangat I, et al. Canadian Registry of implantable electronic device outcomes: surveillance of the Riata lead under advisory. Circ Arrhythm Electrophysiol 2016; 9: pii: e004282.

4. Palamà Z, Trotta R, Mandurino C, et al. Could externalized St. Jude Medical Riata ${ }^{\circledR}$ lead be a culture medium of a polymicrobial endocarditis? A clinical case. Case Rep Cardiol 2017; 2017: 8967234.

5. Polewczyk A, Jachec W, Tomaszewski A, et al. Lead-related infective endocarditis: factors influencing the formation of large vegetations. Europace 2017; 19: 1022-30.

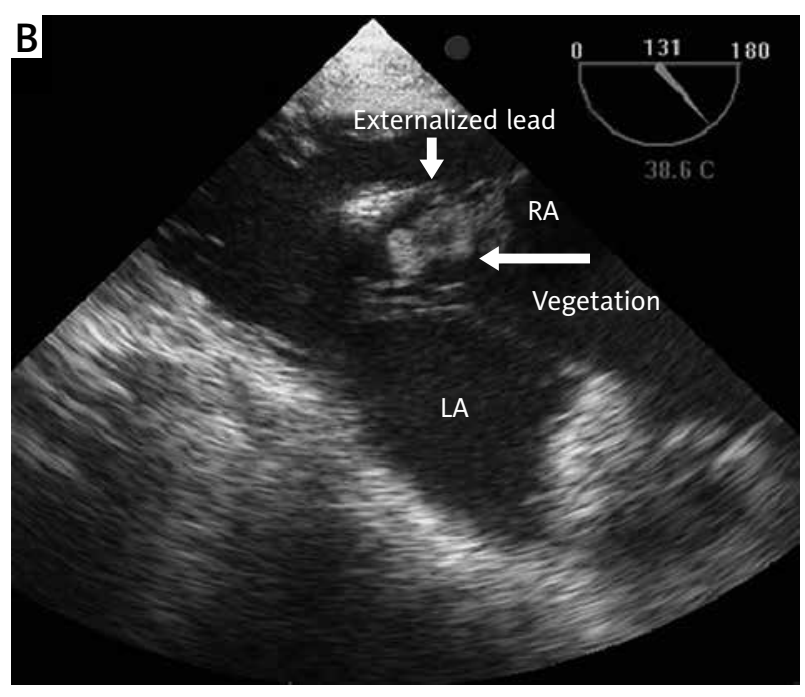

Figure 1. A - Transvenous lead extraction procedure with pulmonary vascular bed protection, B - TEE image showing a large vegetation, $\mathbf{C}-$ externalization of a lead 